\title{
Asymptotic Tracking Control for a Class of Nonlinear Systems with Unknown Failures of Hysteretic Actuators
}

\author{
Chuanjing Hou, ${ }^{1}$ Lisheng $\mathrm{Hu},{ }^{1}$ and Yingwei Zhang ${ }^{2}$ \\ ${ }^{1}$ Department of Automation, Shanghai Jiao Tong University and Key Laboratory of System Control and Information Processing, \\ Ministry of Education of China, Shanghai 200240, China \\ ${ }^{2}$ Key Laboratory of Synthetical Automation for Process Industry, Ministry of Education and Northeastern University, \\ Shenyang, Liaoning 110004, China \\ Correspondence should be addressed to Lisheng Hu; lshu@situ.edu.cn and Yingwei Zhang; zhangyingwei@mail.neu.edu.cn
}

Received 27 May 2014; Accepted 3 June 2014

Academic Editor: Ligang Wu

Copyright (C) 2015 Chuanjing Hou et al. This is an open access article distributed under the Creative Commons Attribution License, which permits unrestricted use, distribution, and reproduction in any medium, provided the original work is properly cited.

An adaptive failure compensation controller for a class of nonlinear systems preceded by hysteretic actuators is proposed in this paper. Three types of high-gain functions are constructed to counteract the effects of the hysteresis, bounded modeling errors, and bounded disturbances. It is shown that the proposed controller not only ensures bounded signals and asymptotic tracking but also avoids possible chattering, despite the presence of unknown hysteretic actuator failures. Simulation results verify the desired failure compensation performance.

\section{Introduction}

The hysteresis phenomenon occurs in all the smart materialbased actuators and sensors. With such nonlinearity in control systems, it may lead to undesirable inaccuracies or oscillations. On the other hand, actuator failures seem inevitable in practical systems. Thus, failure compensation of hysteretic actuators is an important and challenging problem. Adaptive failure compensation has received great attention in recent years [1-12]. However, available results based on adaptive approaches to address hysteretic actuator failures are very limited [13-16].

To address such a challenging problem, it is important to find an appropriate model for the hysteresis. As mentioned in [17], several models were proposed such as Duhen model, Preisach model, Prandtl-Ishlinskii hysteresis operator, BoucWen differential model $[17,18]$. The Bouc-Wen differential model is one of the most widely accepted models of the hysteresis. Actually, it can be shown that the hysteresis model presented in [13-16] is a special case of the Bouc-Wen hysteresis model.

In [13], an adaptive failure compensation scheme for a class of nonlinear systems was studied, where control gains are constants. To avoid possible chattering, the sign (.) functions were involved in the backstepping controller. When the control gains are nonlinear functions of system states, the effects of the actuator hysteresis can no longer be assumed bounded as in [13]. How to handle such effects is a challenging issue especially when there are possible actuator failures. In $[14,15]$, the idea is to separate such effects into two parts by applying Young's inequality. It is noted that the control methods in $[14,15]$ are very complicated and the tracking error cannot asymptotically converge to zero but to the so-called predefined bound. Furthermore, the existence of the estimators increases the order of the closed-loop system.

In this paper, we develop a backstepping $[19,20]$ adaptive compensation controller for a class of nonlinear systems preceded by hysteretic actuators described by Bouc-Wen model. Three types of high-gain functions are incorporated into the controller to counteract the effects of the hysteresis, bounded modeling errors, and bounded disturbances, respectively. In our design, the sign (.) function and a priori knowledge on the bounds of control gains are not required. Besides showing the stability of the closed-loop system, the tracking error is also ensured to achieve zero asymptotically. 
The rest of the paper is outlined as follows. In Section 2, the control problem is formulated. In Section 3, a robust adaptive compensation scheme with high-gain functions is proposed. In Section 4, the stability analysis is presented. Simulation results are presented to show the proposed scheme is effective in Section 5. Finally, this paper is concluded in Section 6.

\section{Problem Formulation}

Consider a class of nonlinear systems in the following form [15]:

$$
\begin{aligned}
\dot{x}_{i} & =x_{i+1}+a^{T} \varphi_{i}\left(\bar{x}_{i}\right) \quad(i=1,2, \ldots, \rho-1) \\
& \vdots \\
\dot{x}_{\rho} & =\varphi_{0}(x, \xi)+a^{T} \varphi_{\rho}\left(\bar{x}_{\rho}\right)+\sum_{j=1}^{m} b_{j} \beta_{j}(x) u_{j}+d(t)+\eta(x, t) \\
\dot{\xi} & =\psi(x, \xi)+a^{T} \phi(x, \xi), \\
y & =x_{1},
\end{aligned}
$$

where $\rho$ is the relative degree of the system; $u_{j} \in R, j=$ $1,2, \ldots, m$ are the inputs whose actuators may fail during system operation; $\bar{x}_{i}=\left[x_{1}, x_{2}, \ldots, x_{i}\right]^{T}(i=1, \ldots, n-1)$, $x=\left[x_{1}, x_{2}, \ldots, x_{n}\right]^{T}$ are the state vectors; $a=\left[a_{1}, \ldots, a_{q}\right]^{T}$ are unknown constant parameters; $b_{j} \in R(j=1,2, \ldots, m)$ are unknown constant parameters with known signs; $\psi_{0} \in$ $R, \varphi_{j} \in R^{\rho}(j=1, \ldots, \rho), \beta_{j}(x) \neq 0 \in R(j=$ $1, \ldots, m), \psi \in R^{n-\rho}$, and $\phi \in R^{(n-\rho) \times \rho}$ are known smooth functions; $|d(t)| \leq \bar{D}$ denotes bounded disturbance; and $\eta(x, t)$ is an unknown nonlinear function representing system modeling errors. There exists a known function $\delta(x, t)$ such that $|\eta(x, t)| \leq \delta(x, t)$.

The hysteresis nonlinearity can be described by BoucWen model $[17,18]$. Consider

$$
\begin{aligned}
& v_{i}=\mu_{i} k_{i} u_{c i}+\left(1-\mu_{i}\right) k_{i} \zeta_{i}=\mu_{i 1} u_{c i}+\mu_{i 2} \zeta_{i} \\
& \dot{\zeta}_{i}=\dot{u}_{c i}-\chi_{i 1}\left|\dot{u}_{c i}\right|\left|\zeta_{i}\right|^{L_{i}-1}-\chi_{i 2} \dot{u}_{c i}\left|\zeta_{i}\right|^{L_{i}},
\end{aligned}
$$

where $0<\mu_{i}<1(i=1, \ldots, m)$ are weighting parameters, $k_{i}(i=1, \ldots, m)$ are stiffness coefficients, $\mu_{i 1}=\mu_{i} k_{i}, \mu_{i 2}=$ $\left(1-\mu_{i}\right) k_{i}(i=1, \ldots, m)$ are constants; $u_{c i}$ is the input of the $i$ th $(i=1, \ldots, m)$ actuator, $\chi_{i 1}, \chi_{i 2}(i=1, \ldots, m)$ describe the shape and amplitude of the $i$ th hysteresis, respectively, $L_{i}$ governs the smoothness of the transition from initial slope to the slope of the asymptote, and $\chi_{i 1} \geq\left|\chi_{i 2}\right|, L_{i} \geq$ $1(i=1,2 \ldots, m)$. By Lemma 1 in [17], $\zeta_{i}(i=1,2 \ldots, m)$ are bounded.

The actuator failure can be modeled as $[8,9,13-15]$

$$
u_{i}=\rho_{i} \mu_{i 1} v_{i}+d_{i}+u_{k i}, \quad \forall t \geq t_{i F}, \quad\left(\rho_{i} u_{k i}=0, i=1, \ldots, m\right),
$$

where $\rho_{i} \in[0,1], u_{k i}$, and $t_{i F}$ are all unknown constants and $d_{i}=\rho_{i} \mu_{i 2} \zeta_{i}(i=1,2 \ldots, m)$ are bounded. For different values of $\rho_{i}$, three types of failures are included:
(1) $\rho_{i}=1$, where the actuator works normally; namely, $u_{i}=u_{c i}$, which is regarded as a failure-free actuator;

(2) $0<\rho_{i}<1$; it implies $u_{i}=\rho_{i} u_{c i}$; the $i$ th actuator is called partial loss of effectiveness (PLOE);

(3) $\rho_{i}=0$; it indicates $u_{i}=u_{k i}$; the $i$ th actuator is called total loss of effectiveness (TLOE).

Remark 1. The values of $\rho_{i}$ can change only from $\rho_{i}=1$ to some values with $0 \leq \rho_{i}<1$. This means that possible changes from normal to any one of the failure cases are unidirectional. The uniqueness of $t_{i F}$ indicates that a failure occurs only once on the $i$ th actuator.

Substituting (2), (3) into (1), we have

$$
\begin{aligned}
\dot{x}_{i}= & x_{i+1}+a^{T} \varphi_{i}\left(\bar{x}_{i}\right) \quad(i=1,2, \ldots, \rho-1) . \\
& \vdots \\
\dot{x}_{\rho}= & \varphi_{0}(x, \xi)+a^{T} \varphi_{\rho}\left(\bar{x}_{\rho}\right) \\
& +\sum_{j=1}^{m} b_{j} \beta_{j}(x)\left(\rho_{j} \mu_{j 1} u_{c j}+u_{k j}+d_{j}\right) \\
& \quad+d(t)+\eta(x, t) \\
\dot{\xi}= & \psi(x, \xi)+a^{T} \phi(x, \xi) \\
y= & x_{1} .
\end{aligned}
$$

To derive a suitable adaptive control scheme, the following assumptions are made.

Assumption 2. When TOLE type of actuator failures up to $m-$ 1 , the remaining actuators can still achieve a desired control objective.

Remark 3. Note that all actuators are allowed to have partial loss of effectiveness simultaneously.

Assumption 4. The zero dynamics of $\dot{\xi}=\psi(x, \xi)+a^{T} \phi(x, \xi)$ is input to state stable with respect to $x$ as its input.

Let $T_{0}=0$. Suppose that there are $p_{k}\left(0 \leq p_{k} \leq m\right)$ actuators failing at time instants $t_{k}, k=1,2, \ldots, q$, and $t_{0}<t_{1}<\cdots t_{q}<\infty$. In other words, all actuators work normally in time interval $\left[t_{0}, t_{1}\right)$ and no new failure will occur after time $t_{q}$. Let the set $Q_{j T}$ denote the actuators of total failure in interval $\left[t_{j}, t_{j+1}\right)$ and use the set $\bar{Q}_{j T}$ to represent other normal actuators. It can be concluded that $Q_{j T} \cup \bar{Q}_{j T}=$ $\{1,2, \ldots, m\}$.

Our objective is to design a control law $u_{c i}(t)$ for the nonlinear systems with $p$ unknown actuator failures, when $p$ changes at time instants $t_{k}, k=1,2, \ldots, q$, such that the output $y(t)$ asymptotically tracks a given reference signal $y_{r}(t)$ with up to $\rho$ th order derivatives bounded and that all closed-loop signals are bounded. 


\section{Adaptive Compensation Control Schemes}

The backstepping technique $[19,20]$ is applied to derive an adaptive actuator failure compensation controller. The following change of coordinates is required:

$$
\begin{aligned}
& z_{1}=x_{1}-y_{r} \\
& z_{i}=x_{i}-y_{r}^{(i-1)}-\alpha_{i-1} \quad(i=2, \ldots, \rho),
\end{aligned}
$$

where $z_{1}$ is the tracking error and $\alpha_{\rho}$ is the $\rho$ th stabilizing function. To illustrate the backstepping procedures, only the last step of the design is elaborated in details.

Step $i$. Consider $i=1, \ldots, \rho-1$; the $i$ th stabilizing function $\alpha_{i}$, the $i$ th regressor $\omega_{i}$, and the $i$ th tuning function $\tau_{i}$ are chosen as

$$
\begin{aligned}
\alpha_{1}= & -c_{1} z_{1}-\varphi_{1}\left(x_{1}\right)^{T} \widehat{a} \\
\alpha_{i}= & -z_{i-1}-c_{i} z_{i}-\omega_{i}^{T} \widehat{a} \\
& +\sum_{k=1}^{i-1}\left(\frac{\partial \alpha_{i-1}}{\partial x_{k}} x_{k+1}+\frac{\partial \alpha_{i-1}}{\partial y_{r}^{(k-1)}} y_{r}^{(k)}\right) \\
& +\frac{\partial \alpha_{i-1}}{\partial \widehat{a}} \Gamma \tau_{i}+\sum_{k=2}^{i-1}\left(\frac{\partial \alpha_{k-1}}{\partial \widehat{a}} \Gamma \omega_{i} z_{k}\right), \quad(i=2, \ldots, \rho-1) \\
\omega_{i}= & \varphi_{i}-\sum_{k=1}^{i-1} \frac{\partial \alpha_{i-1}}{\partial x_{k}} \varphi_{k} \quad(i=1, \ldots, \rho-1) \\
\tau_{i}= & \tau_{i-1}+\omega_{i} z_{i} \quad(i=1, \ldots, \rho-1),
\end{aligned}
$$

where $c_{i}(i=1, \ldots, \rho-1)$ are positive design parameters and $\widehat{a} \in R^{q}$ is estimator of the unknown vector $a$.

Step $\rho$. From (6), the derivative of $z_{\rho}$ is

$$
\begin{aligned}
\dot{z}_{\rho}= & \varphi_{0}(x, \xi)+\varphi_{\rho}\left(\bar{x}_{\rho}\right)^{T} a \\
& +\sum_{i=1}^{m} b_{i} \beta_{i}(x)\left(\rho_{i} \mu_{i 1} u_{c i}+u_{k i}+d_{i}\right)+\eta(x, t) \\
& +d(t)-\sum_{k=1}^{\rho-1}\left(\frac{\partial \alpha_{\rho-1}}{\partial x_{k}} x_{k+1}+\varphi_{k}^{T} a\right) \\
& -\frac{\partial \alpha_{\rho-1}}{\partial \widehat{a}} \dot{a}-\sum_{k=1}^{\rho-1}\left(\frac{\partial \alpha_{\rho-1}}{\partial y_{r}^{(k-1)}} y_{r}^{(k)}\right)-y_{r}^{(\rho)} .
\end{aligned}
$$

If the effects of hysteresis are treated as disturbances, it should be noted that the disturbances in (7) can be classified into three types: (1) $d(t)$ is bounded by an unknown constant $D$; (2) $\eta(x, t)$ is bounded by a known function; (3) $\sum_{j=1}^{m} b_{j} \beta_{j}(x) d_{i}$ cannot be bounded by any known function but $\beta_{j}(x)(j=1, \ldots, m)$ are known and $b_{j} d_{j}(j=1,2 \ldots, m)$ are bounded. According to their different characteristics, three high-gain functions will be proposed to counteract the effects of the disturbances. At the final step, the stabilizing function $\alpha_{\rho}$ is given by

$$
\begin{aligned}
\alpha_{\rho}= & -\varphi_{0}-\omega_{\rho}^{T} \widehat{a}-z_{\rho-1}-c_{\rho} z_{\rho}+y_{r}^{(\rho)} \\
& +\sum_{k=1}^{\rho-1}\left(\frac{\partial \alpha_{\rho-1}}{\partial x_{k}} x_{k+1}+\frac{\partial \alpha_{\rho-1}}{\partial y_{r}^{(k-1)}} y_{r}^{(k)}\right) \\
& +\frac{\partial \alpha_{\rho-1}}{\partial \widehat{a}} \Gamma \tau_{\rho}-e^{f(t)} z_{\rho}-e^{f(t)} \delta(x)^{2} z_{\rho}-e^{f(t)} \sum_{k=1}^{m} \beta_{i}^{2}(x) z_{\rho},
\end{aligned}
$$

where

$$
\begin{aligned}
& \omega_{\rho}=\varphi_{\rho}-\sum_{k=1}^{\rho-1} \frac{\partial \alpha_{\rho-1}}{\partial x_{k}} \varphi_{k} \\
& \tau_{\rho}=\tau_{\rho-1}+\omega_{\rho} z_{\rho} .
\end{aligned}
$$

$f(t)$ is bounded and $\lim _{t \rightarrow \infty} \int_{0}^{t} e^{-f(\tau)} d \tau$ exists, and $c_{\rho}$ is a positive design parameter.

Let

$$
\omega=\left[\alpha_{\rho}, \beta_{1}, \ldots, \beta_{m}\right]^{T} .
$$

The control law and parameter update laws are obtained as follows

$$
\begin{aligned}
u_{c i} & =\operatorname{sign}\left(b_{i}\right) \frac{1}{\beta_{i}(x)} \widehat{k}^{T} \omega, \quad(i=1, \ldots, m) \\
\dot{\hat{a}} & =\Gamma \tau_{\rho} \\
\dot{\hat{k}} & =-\Gamma_{k} \omega z_{\rho},
\end{aligned}
$$

where $\widehat{k}=\left[\widehat{k}_{1}, \widehat{k}_{2}^{T}\right]^{T}, \widehat{k}_{2}=\left[\widehat{k}_{21}, \widehat{k}_{22}^{T}, \ldots, \widehat{k}_{2 m}^{T}\right]^{T}, \widehat{k}$ and $\widehat{a}$ are the estimates of $k$ and $a$, respectively, and $\Gamma, \Gamma_{k}$ are positive definite matrices chosen by users. If $k$ is a desired constant vector which can be chosen to satisfy

$$
\sum_{i=1, i \notin \overline{\mathbb{Q}}_{j T}}^{m}\left|b_{i}\right| \rho_{i} k^{T} w=\alpha_{\rho}-\sum_{j \in Q_{j T}} b_{j} \beta_{j} u_{k j}
$$

this gives

$$
k_{1}=\frac{1}{\sum_{i=1, i \notin \bar{Q}_{j T}}^{m}\left|b_{i}\right| \rho_{i}}, \quad k_{2, j}=-\frac{b_{j} u_{k j}}{\sum_{i=1, i \notin \bar{Q}_{j T}}^{m}\left|b_{i}\right| \rho_{i}} .
$$

Substituting (11)-(13) and (8) into (7), we have

$$
\begin{aligned}
\dot{z}_{\rho}= & -z_{\rho-1}-c_{\rho} z_{\rho}+\omega_{\rho}^{T} \widetilde{a} \\
& +\sum_{i \in \bar{Q}_{j T}}\left|b_{i}\right| \rho_{i} \mu_{i 1} \widetilde{k}^{T} \omega-\left(e^{f(t)} z_{\rho}-d(t)\right) \\
& -\left(e^{f(t)} \delta(x)^{2} z_{\rho}-\eta(x, t)\right) \\
& -\left(e^{f(t)} \sum_{k=1}^{m} \beta_{k}^{2}(x) z_{\rho}-\sum_{k=1}^{m} \beta_{k}(x) b_{k} d_{k}\right) .
\end{aligned}
$$




\section{Stability Analysis}

To prepare for the stability analysis, we rewrite the error system as

$$
\dot{z}=A_{z}(z, t) z+W_{\theta}(z, t)^{T} \tilde{a}+D(t) e_{\rho}
$$

where the system matrices $A_{z}(z, t), W_{\theta}(z, t), D(t)$, and $e_{\rho}$ are given by

$$
\begin{aligned}
& A_{z}(z, t) \\
& =\left[\begin{array}{cccccc}
-c_{1} & 1 & 0 & \cdots & \cdots & 0 \\
1 & -c_{2} & 1+\sigma_{23} & \sigma_{24} & \cdots & \sigma_{2 \rho} \\
\vdots & -1-\sigma_{23} & \ddots & \ddots & \ddots & \vdots \\
\vdots & -\sigma_{24} & \ddots & \ddots & \ddots & \sigma_{\rho-2 \rho} \\
\vdots & \vdots & \ddots & \ddots & \ddots & 1+\sigma_{\rho-1, \rho} \\
0 & -\sigma_{2 \rho} & \cdots & -\sigma_{\rho-2, \rho} & -1-\sigma_{\rho-1, \rho} & -c_{\rho}
\end{array}\right] \\
& W_{\varepsilon}(z, t)=\left[\omega_{1} \omega_{2}, \ldots, \omega_{\rho}\right]^{T} \in R^{\rho} \text {. } \\
& W_{\theta}(z, t)=W_{\varepsilon}(z, t) \omega^{T}-\hat{\rho}\left(\dot{y}_{r}+\bar{\alpha}_{1}\right) e_{1} e_{1}^{T} \in R^{\rho \times \rho} \\
& D(t)=\sum_{i \in \overline{\mathrm{Q}}_{j T}}\left|b_{i}\right| \rho_{i} \mu_{i 1} \widetilde{k}^{T} \omega-\left(e^{f(t)} z_{\rho}-d(t)\right) \\
& -\left(e^{f(t)} \delta(x)^{2} z_{\rho}-\eta(x, t)\right) \\
& e_{\rho}=[0, \ldots, 0,1]^{T} \in R^{\rho} \\
& \sigma_{j k}=-\frac{\partial \alpha_{j-1}}{\partial \widehat{a}} \Gamma \omega_{k}
\end{aligned}
$$
ties.

The closed-loop system has the following desired proper-

Theorem 5. With the ISS of the zero dynamics of system (1) and $m$ hysteretic actuators modeled in (2) with possible unknown failures by (3), the controller (11) with the adaptive laws (12) and (13) ensures the boundedness of the closed-loop signals and the asymptotic output tracking: $\lim _{t \rightarrow \infty}\left(y-y_{r}\right) \rightarrow$ 0 .

Proof. For each time interval $\left(t_{k}, t_{k+1}\right), k=0,1,2, \ldots, q$, we have a Lyapunov function $V$ in the following form:

$$
V=\frac{1}{2} \sum_{i=1}^{\rho} z_{i}^{2}+\frac{1}{2} \widetilde{a}^{T} \Gamma \widetilde{a}+\sum_{i \in \bar{Q}_{j T}} \frac{\rho_{i} \mu_{i 1}\left|b_{i}\right|}{2} \widetilde{k}_{j}^{T} \Gamma_{k} \widetilde{k}_{j}
$$

Taking the derivative of (19) yields

$$
\dot{V}=\sum_{i=1}^{\rho} z_{i} \dot{z}_{i}-\tilde{a}^{T} \Gamma \dot{\hat{a}}-\sum_{i \in \bar{Q}_{j T}} \rho_{i} \mu_{i 1}\left|b_{i}\right| \widetilde{k}_{j}^{T} \Gamma_{k} \dot{\hat{k}}_{j} .
$$

Substituting (11)-(13) and (8) into (7), we have

$$
\begin{aligned}
\dot{V}= & -\sum_{i=1}^{\rho} c_{i} z_{i}^{2}-\left(e^{f(t)} z_{\rho}^{2}-d(t) z_{\rho}\right) \\
& -\left(e^{f(t)} \delta(x)^{2} z_{\rho}^{2}-\eta(x, t) z_{\rho}\right) \\
& -\left(e^{f(t)} \sum_{k=1}^{m} \beta_{k}^{2}(x) z_{\rho}^{2}-\sum_{k=1}^{m} \beta_{k}(x) b_{k} d_{k} z_{\rho}\right) .
\end{aligned}
$$

From $|d(t)| \leq \bar{D},|\eta(x, t)| \leq \delta(x, t)$, and bounded $d_{i}(i=$ $1,2, \ldots, m)$, we get

$$
\begin{aligned}
\dot{V} \leq & -\sum_{i=1}^{\rho} c_{i} z_{i}^{2}-\left(e^{f(t)} z_{\rho}^{2}-|\bar{D}|\left|z_{\rho}\right|\right) \\
& -\left(e^{f(t)} \delta(x)^{2} z_{\rho}^{2}-|\delta(x)|\left|z_{\rho}\right|\right) \\
& -\left(e^{f(t)} \sum_{k=1}^{m} \beta_{k}^{2}(x) z_{\rho}^{2}-\sum_{k=1}^{m}\left|\beta_{k}(x)\right|\left|b_{k} d_{k}\right|\left|z_{\rho}\right|\right) \\
\leq & -\sum_{i=1}^{\rho} c_{i} z_{i}^{2}+\frac{\bar{D}^{2}}{4} e^{-f(t)}+\frac{1}{4} e^{-f(t)}+\frac{1}{4} \sum_{k=1}^{m}\left|b_{k}^{2} d_{k}^{2}\right| e^{-f(t)} \\
= & -\sum_{i=1}^{\rho} c_{i} z_{i}^{2}+\frac{\sum_{k=1}^{m}\left|b_{k}^{2} d_{k}^{2}\right|+\bar{D}^{2}+1}{4} e^{-f(t)} \\
= & -\sum_{i=1}^{\rho} c_{i} z_{i}^{2}+D_{0} e^{-f(t)},
\end{aligned}
$$

where

$$
D_{0}=\frac{\sum_{k=1}^{m}\left|b_{k}^{2} d_{k}^{2}\right|+\bar{D}^{2}+1}{4} .
$$

From (22), we conclude that $V(t) \in L_{\infty}, t \in\left[t_{0}, t_{1}\right)$, so that $z_{i}(i=1, \ldots, \rho), \dot{z}_{i}(i=1, \ldots, \rho)$, and $\widetilde{a}, \widetilde{k}$ are bounded for $t \in\left[t_{0}, t_{1}\right)$. It follows from (8) and (10) that $\alpha_{\rho}$ and $\omega$ are bounded. Therefore, all closed-loop signals are bounded for $\left(t_{0}, t_{1}\right)$. In order to prove the asymptotic tracking, considering the last time interval $\left(t_{q}, \infty\right)$. From $(22)$, we can obtain

$$
c_{i} \int_{0}^{t} z_{i}^{2}(\tau) d \tau \leq V(0)-V(t)+D_{0} \int_{0}^{t} e^{-f(\tau)} d \tau .
$$

Because of the boundedness of $D_{0} \int_{0}^{t} e^{-f(\tau)} d \tau$, we have $z_{i} \in L_{2},(i=1, \ldots, \rho)$. By Barbalat's lemma, $\lim _{t \rightarrow \infty}\left(z_{i}(t)\right) \rightarrow 0(i=1, \ldots, \rho)$ can be obtained. This completes the proof.

\section{Simulations}

We consider a second order nonlinear [15] system with two inputs described as

$$
\begin{aligned}
& \dot{x}_{1}=x_{2}+\varphi_{1}^{T}\left(x_{1}\right) \theta \\
& \dot{x}_{2}=\varphi_{2}^{T}(x) \theta+\sum_{i=1}^{2} b_{i} \beta_{i}(x) u_{i}+\eta(x, t),
\end{aligned}
$$



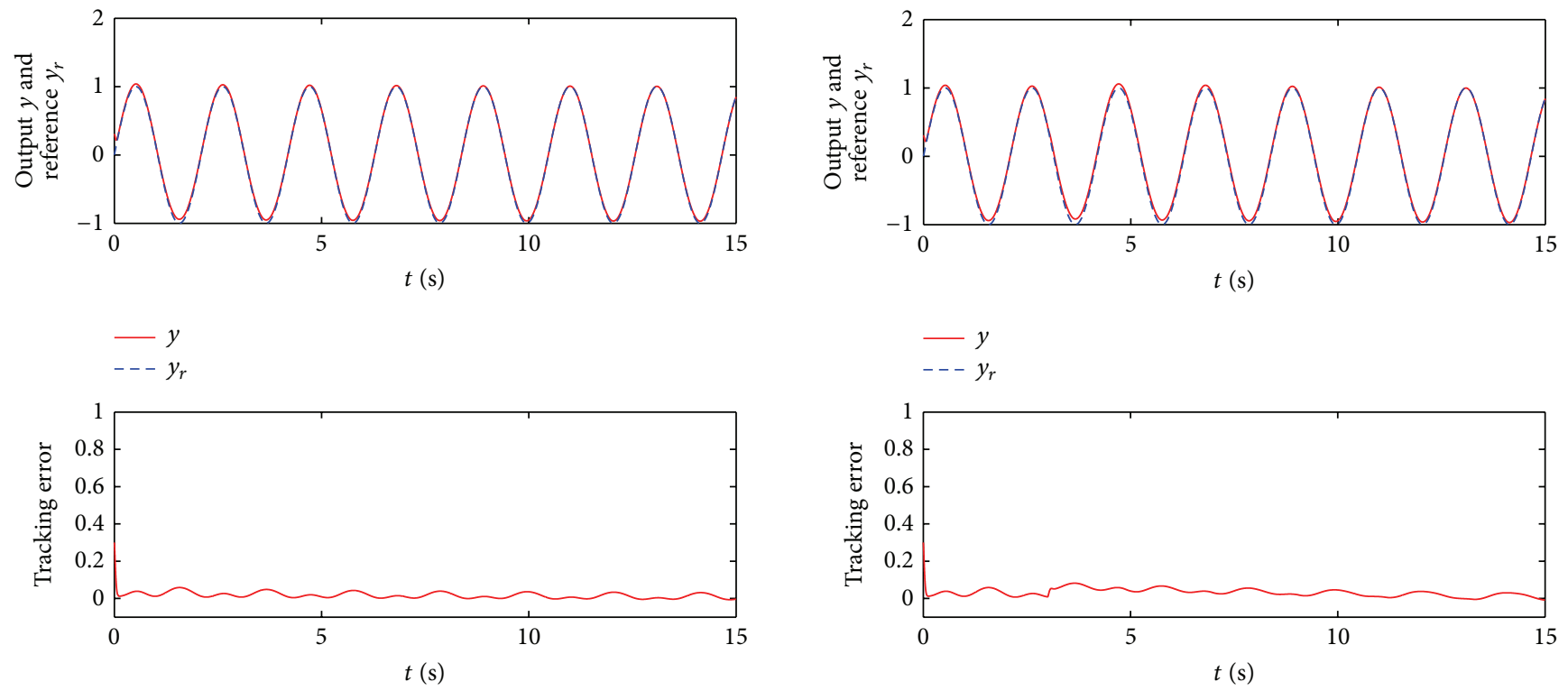

FIgURE 1: Output $y$, reference $y_{r}$, and error $e(t)$.

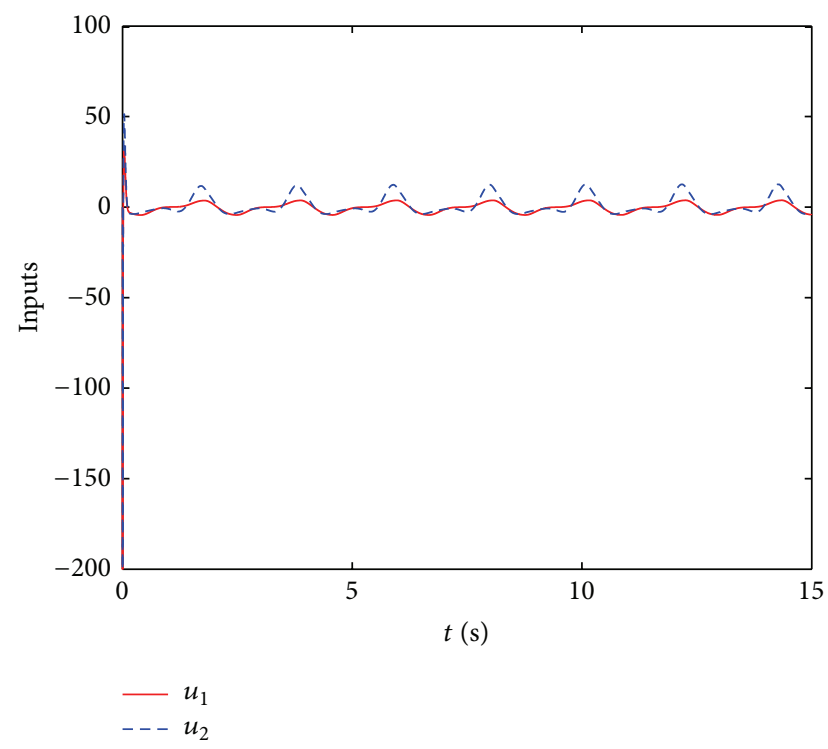

FIGURE $2: u_{1}$ and $u_{2}$.

where $\varphi_{1}\left(x_{1}\right)=1+x_{1}^{2}, \varphi_{2}(x)=1+\sin \left(x_{1}\right), \beta_{1}(x)=1.9+$ $0.1 \sin x_{1}, \beta_{2}(x)=e^{x_{1}}, \theta=2, b_{1}=b_{2}=1, b_{1}, b_{2}$ are unknown constants, $u_{1}, u_{2}$ are the outputs of two hysteretic actuators, $x_{1}, x_{2}$ are the states, and $\eta(x)=0.1 x_{1}^{2}(1+\sin t)\left(1+\sin x_{2}\right)$ is bounded by $\delta(x)=0.4 x_{1}^{2}$. The reference signal is set as $y_{r}(t)=\sin (3 t)$. The backlash-like hysteresis is described by (2) with parameters $\mu_{11}=\mu_{21}=\mu_{12}=\mu_{22}=1, \chi_{11}=\chi_{21}=1$, $\chi_{12}=\chi_{22}=0$, and $L_{1}=L_{2}=1$. The high-gain function is chosen as $f(t)=e^{2.5 \arctan (t)}$. For simulation, we consider three actuator failure cases.

Case 1. There are no actuator failures.

By Theorem 5, we can obtain the actual control law and the update laws. The initial conditions are set as follows:

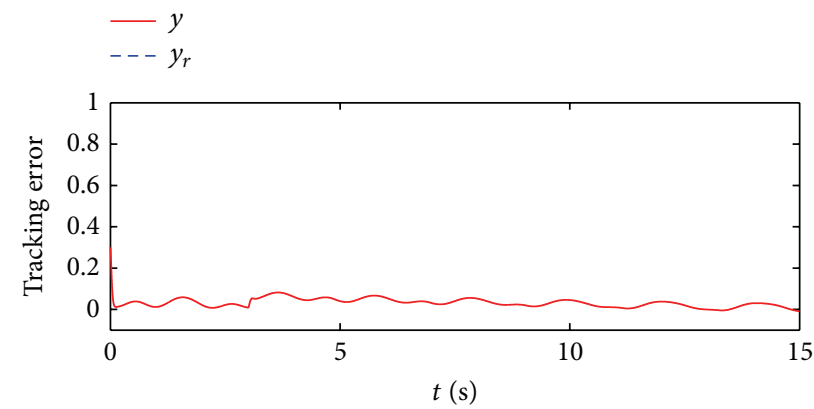

FIgURE 3: Output $y$, reference $y_{r}$, and error $e(t)$.

$$
\begin{gathered}
\widehat{a}=1.5, \quad \Gamma=0.0005, \\
\widehat{k}=[0.4,0,0]^{T}, \quad \Gamma_{k}=0.0001 * I_{3} \\
x_{0}=[0.3,0]^{T}, \quad c_{1}=60, \quad c_{2}=60,
\end{gathered}
$$

where $I_{3}$ is the 3 rd order identity matrix.

The simulation results including output $y(t)$, reference output $y_{r}(t)$, and tracking error $e(t)$ are shown in Figure 1; the actuators outputs $u_{1}, u_{2}$ are shown in Figure 2. The system responses are as expected. At the beginning, there is a transient response in tracking errors. But, as time goes on, the tracking errors become smaller and ultimately vanish. The proposed controller guarantees that asymptotic tracking is achieved.

Case 2. Actuator $u_{1}$ is stuck at $u_{1}=45$ from $t=3 \mathrm{~s}$, thus undergoing a TLOE type of failure. By Theorem 5 , we can obtain the actual control law and the update laws. The initial conditions are set as follows.

The other parameters are the same as those in Case 1.

The simulation results including output $y(t)$, reference output $y_{r}(t)$, and tracking error $e(t)$ are shown in Figure 3; the actuators outputs $u_{1}, u_{2}$ are shown in Figure 4 . The system responses are as expected. When one of the actuators fails, there is a transient response in tracking errors. But, as time goes on, the tracking errors become smaller and ultimately vanish. The proposed controller guarantees that asymptotic tracking is achieved.

Case 3. Actuator $u_{2}$ is stuck at $u_{2}=40$ from $t=3 \mathrm{~s}$ and actuator $u_{1}$ loses $60 \%$ from $t=6 \mathrm{~s}$. Thus, $u_{1}$ undergoes a PLOE type of failure while $u_{2}$ is a TLOE type of failure.

The other parameters are the same as those in Case 1. 


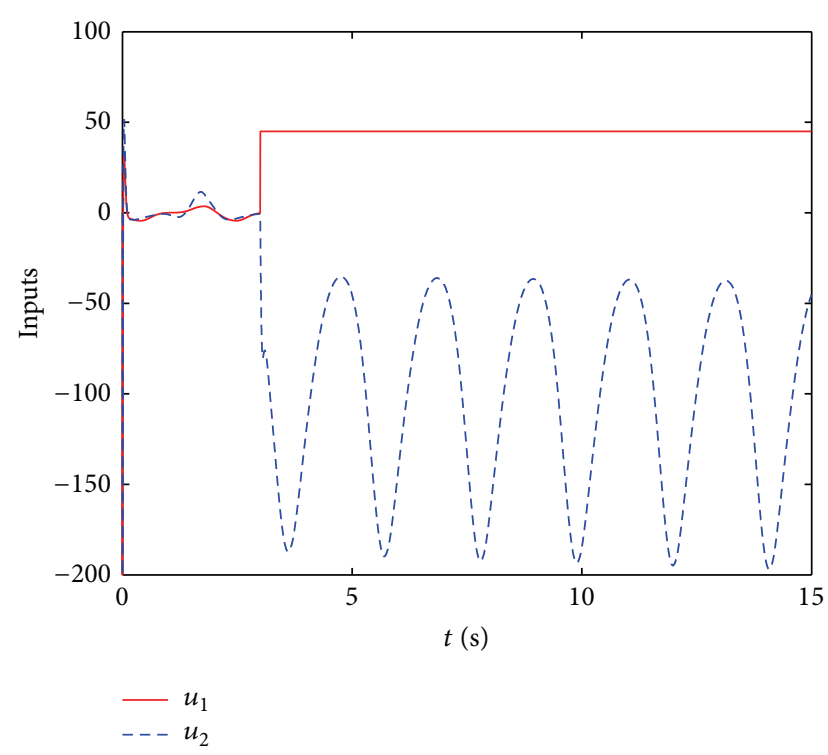

FIGURE $4: u_{1}$ and $u_{2}$.


FIGURE 5: Output $y$, reference $y_{r}$, and error $e(t)$.

The simulation results including output $y(t)$, reference output $y_{r}(t)$, and tracking error $e(t)$ are shown in Figure 5; the actuators outputs $u_{1}, u_{2}$ are shown in Figure 6 . The system responses are as expected. When the actuators fail, there is a transient response in tracking errors. But, as time goes on, the tracking errors become smaller and ultimately vanish. The proposed controller guarantees that asymptotic tracking is achieved.

\section{Conclusions}

This paper presents an adaptive failure compensation controller for a class of uncertain nonlinear systems dominated

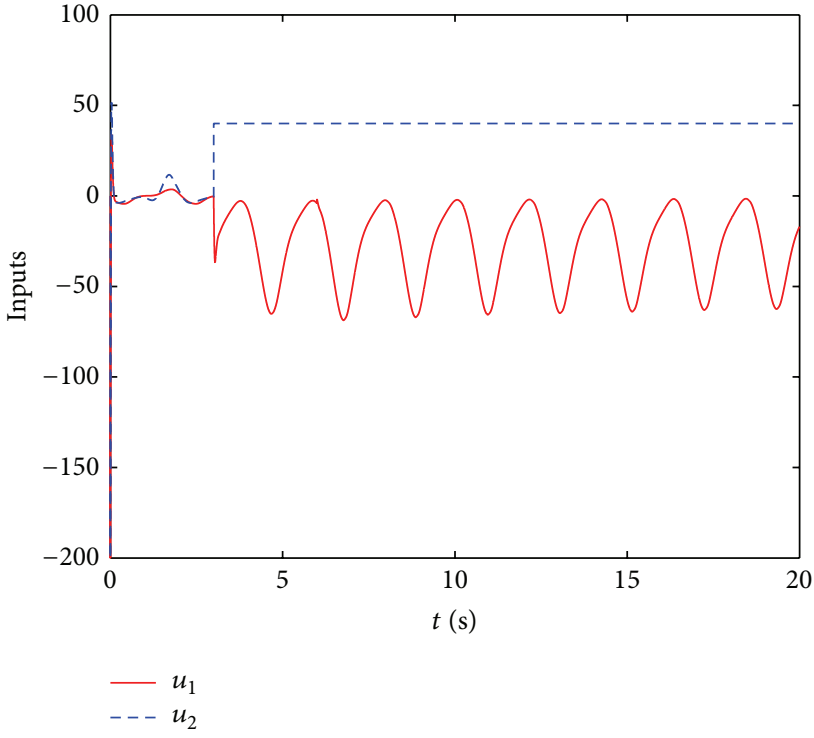

Figure 6: $u_{1}$ and $u_{2}$.

by the hysteresis actuator nonlinearity. We propose three types of high-gain functions to deal with the unknown bounded disturbances, unknown modeling errors, and unknown actuator failures. It has been shown that the tracking errors can converge to zero asymptotically while all the closed-loop signals remain bounded. Furthermore, the proposed scheme can avoid possible chattering. Simulation results illustrate the effectiveness of our proposed scheme.

\section{Conflict of Interests}

The authors declare that there is no conflict of interests regarding the publication of this paper.

\section{References}

[1] G. Tao and P. V. Kokotovic, Adaptive Control of Systems with Actuator and Sensor Nonlinearities, John Wiley \& Sons, New York, NY, USA, 1996.

[2] G. Tao, S. Chen, and S. M. Joshi, "An adaptive control scheme for systems with unknown actuator failures," Automatica, vol. 38, no. 6, pp. 1027-1034, 2002.

[3] X. Tang, G. Tao, and S. M. Joshi, "Adaptive actuator failure compensation for parametric strict feedback systems and an aircraft application," Automatica, vol. 39, no. 11, pp. 1975-1982, 2003.

[4] X. Tang, G. Tao, and S. M. Joshi, "Adaptive output feedback actuator failure compensation for a class of state-dependent nonlinear systems," in Proceedings of the 42nd IEEE Conference on Decision and Control, pp. 1681-1686, Maui, Hawaii, December 2003.

[5] G. Tao, S. Chen, X. D. Tang, and S. M. Joshi, Adaptive Control of Systems with Actuator Failures, Springer, London, UK, 2004.

[6] X. Tang, G. Tao, and S. M. Joshi, "Adaptive output feedback design for actuator failure compensation using dynamic bounding: output tracking and an application," in Proceedings 
of the American Control Conference (ACC '05), pp. 3186-3191, Portland, Ore, USA, June 2005.

[7] X. D. Tang and G. Tao, "An adaptive nonlinear output feedback controller using dynamic bounding with an aircraft control application," International Journal of Adaptive Control and Signal Processing, vol. 23, no. 7, pp. 609-639, 2009.

[8] W. Wang and C. Wen, "Adaptive output feedback controller design for a class of uncertain nonlinear systems with actuator failures," in Proceedings of the 49th IEEE Conference on Decision and Control (CDC '10), pp. 1749-1754, Atlanta, Ga, USA, December 2010.

[9] W. Wang and C. Wen, "Adaptive actuator failure compensation control of uncertain nonlinear systems with guaranteed transient performance," Automatica, vol. 46, no. 12, pp. 2082-2091, 2010.

[10] J. Zhou, C. Wen, and Y. Zhang, "Adaptive backstepping control of a class of uncertain nonlinear systems with unknown backlash-like hysteresis," IEEE Transactions on Automatic Control, vol. 49, no. 10, pp. 1751-1757, 2004.

[11] Z. Zhang and W. Chen, "Adaptive output feedback control of nonlinear systems with actuator failures," Information Sciences. An International Journal, vol. 179, no. 24, pp. 4249-4260, 2009.

[12] Z. Zhang, S. Xu, and B. Wang, "Adaptive actuator failure compensation with unknown control gain signs," IET Control Theory \& Applications, vol. 5, no. 16, pp. 1859-1867, 2011.

[13] J. Cai, C. Wen, H. Su, X. Li, and Z. Liu, "Adaptive failure compensation of hysteric actuators in controlling uncertain nonlinear systems," in Proceedings of the American Control Conference (ACC '11), pp. 2320-2325, San Francisco, Calif, USA, July 2011.

[14] J. Cai, C. Wen, H. Su, Z. Liu, and X. Liu, "Robust adaptive failure compensation of hysteretic actuators for parametric strict feedback systems," in Proceedings of the 50th IEEE Conference on Decision and Control and European Control Conference (CDCECC '11), pp. 7988-7993, December 2011.

[15] J. Cai, C. Wen, H. Su, and Z. Liu, "Robust adaptive failure compensation of hysteretic actuators for a class of uncertain nonlinear systems," IEEE Transactions on Automatic Control, vol. 58, no. 9, pp. 2388-2394, 2013.

[16] J. Zhou and C. Wen, Adaptive Backstepping Control of Uncertain Systems, vol. 372, Springer, Berlin, Germany, 2007.

[17] J. Zhou, C. Wen, and T. Li, "Adaptive output feedback control of uncertain nonlinear systems with hysteresis nonlinearity," IEEE Transactions on Automatic Control, vol. 57, no. 10, pp. 26272633, 2012.

[18] Z. Zhang, S. Xu, and B. Zhang, "Asymptotic tracking control of uncertain nonlinear systems with unknown actuator nonlinearity," IEEE Transactions on Automatic Control, vol. 59, no. 5, pp. 1336-1341, 2014.

[19] M. Krstic, I. Kancellakopoulos, and P. V. Kokotovivc, Nonlinear and Adaptive Control Design, Wiley, New York, NY, USA, 1995.

[20] A. Isidori, Nonlinear Control Systems, Springer, New York, NY, USA, 3rd edition, 1995. 


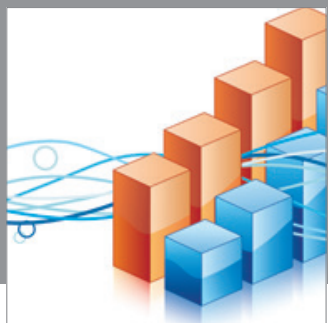

Advances in

Operations Research

mansans

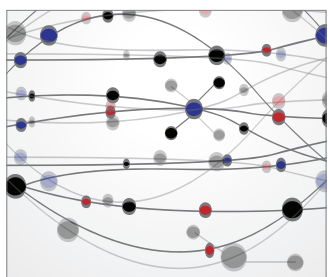

The Scientific World Journal
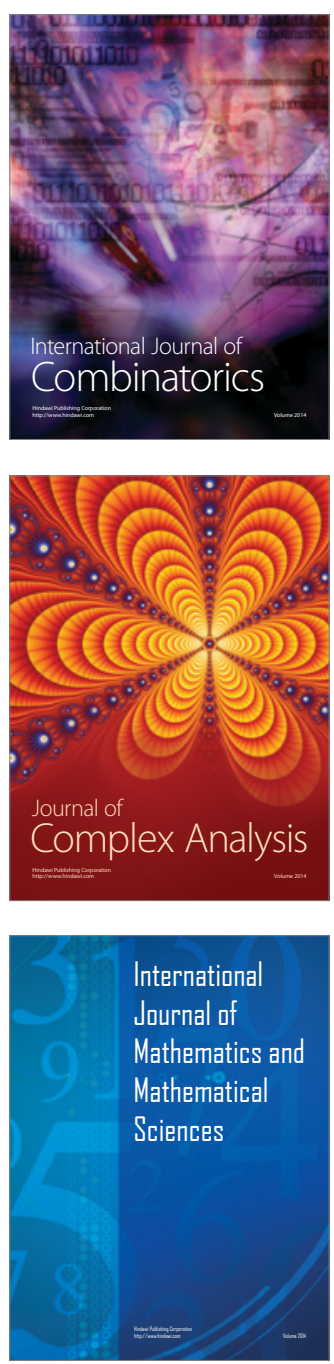


Submit your manuscripts at http://www.hindawi.com


Journal of

Function Spaces

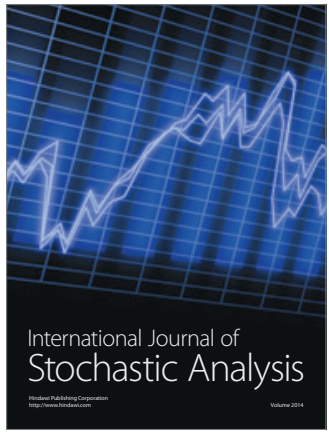

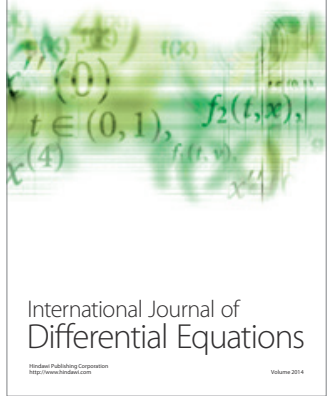
